\title{
Anatomy and clinical significance of the maxillary nerve: a literature review
}

\author{
I.M. Tomaszewska1 ${ }^{1}$, H. Zwinczewska², T. Gładysz ${ }^{3}$, J.A. Walocha² \\ ${ }^{1}$ Department of Medical Education, Jagiellonian University Medical College, Krakow, Poland \\ ${ }^{2}$ Department of Anatomy, Jagiellonian University Medical College, Krakow, Poland \\ ${ }^{3}$ Department of Oral Surgery, Jagiellonian University Medical College, Krakow, Poland \\ [Received 18 July 2014; Accepted 28 October 2014]
}

Background: The aim of this paper was to summarise the anatomical knowledge on the subject of the maxillary nerve and its branches, and to show the clinical usefulness of such information in producing anaesthesia in the region of the maxilla. Materials and methods: A literature search was performed in Pubmed, Scopus, Web of Science and Google Scholar databases, including studies published up to June 2014, with no lower data limit.

Results: The maxillary nerve (V2) is the middle sized branch of the trigeminal nerve - the largest of the cranial nerves. The V2 is a purely sensory nerve supplying the maxillary teeth and gingiva, the adjoining part of the cheek, hard and soft palate mucosa, pharynx, nose, dura mater, skin of temple, face, lower eyelid and conjunctiva, upper lip, labial glands, oral mucosa, mucosa of the maxillary sinus, as well as the mobile part of the nasal septum. The branches of the maxillary nerve can be divided into four groups depending on the place of origin i.e. in the cranium, in the sphenopalatine fossa, in the infraorbital canal, and on the face.

Conclusions: This review summarises the data on the anatomy and variations of the maxillary nerve and its branches. A thorough understanding of the anatomy will allow for careful planning and execution of anaesthesiological and surgical procedures involving the maxillary nerve and its branches. (Folia Morphol 2015; 74, 2: 150-156)

Key words: greater palatine nerve, infraorbital nerve, maxillary nerve, palate, nasopalatine nerve, sphenopalatine ganglion, trigeminal nerve, zygomatic nerve

\section{AIM AND SEARCH METHODOLOGY}

The aim of this paper was to summarise the anatomical knowledge on the subject of the maxillary nerve and its branches, and to show the clinical usefulness of such data in producing anaesthesia in the region of the maxilla. A literature search was performed in Pubmed, Scopus, Web of Science and Google Scholar databases, including studies published up to June 2014, with no lower data limit. Additionally, a manual search of pertinent manuscript references, as well as in the major anatomical, radiological, dental and cranio-maxillo-facial journals was carried out. The importance of studies was determined hierarchically — starting from the title, next the abstract, and finally basing on the full-text manuscript.

\section{THE MAXILLARY NERVE: AN OVERVIEW}

\section{Introduction}

The maxillary nerve (V2) is the middle sized (between the larger mandibular and smaller ophthalmic) branch of the trigeminal nerve $(\mathrm{V})$ - the largest of the

Address for correspondence: I.M. Tomaszewska, DDS, Department of Medical Education, Jagiellonian University Medical College, ul. Łazarza 16, 31-530 Kraków, Poland, tel: +48-666-666-987, e-mail: im.tomaszewska@gmail.com 
cranial nerves. It is a purely sensory nerve supplying the maxillary teeth and gingiva, the adjoining part of the cheek, hard and soft palate mucosa, pharynx, nose, dura mater, skin of temple, face, lower eyelid and conjunctiva, upper lip, labial glands, oral mucosa, mucosa of the maxillary sinus, as well as the mobile part of the nasal septum [14, 33, 41]. In foetal life, the V2 innervates only the structures of the maxillary process. However, in the course of embryological development this area further extends to include also the frontonasal process [12]. The maxillary nerve communicates with the facial nerve, and thus substitutes traditional skeletal muscle proprioception with impulses from mechanoreceptors localised in the skin of the face and oral mucosa $[12,14,33,41]$. It has been shown that most of the proprioceptive axons have their cell bodies in the Gasserian ganglion [20].

The maxillary nerve contains about 50 thousand myelinated fibres [34]. Fifty per cent of these fibres divide into ascending and descending branches before reaching the principal sensory nucleus [20]. Based on clinical studies it is thought that V2 fibres are placed centrally in the spinal tract and do not extend below the medulla oblongata [6]. It has been proven that fibres, regardless of division, terminate throughout the whole nucleus [34]. Fibres of the posterior face terminate in the lower part, whilst those from the upper lip, mouth and the tip of the nose terminate at a higher level. This can give rise to a segmental (crossdivisional) sensory loss seen in syringobulbia — common in tertiary syphilis [34].

\section{Origin and course of the maxillary nerve}

The maxillary nerve, as well as its branches and their course can be seen in Figure 1. The maxillary nerve originates from the trigeminal ganglion (Gasser's or Gasser's ganglion), which is located in Meckel's cave (lat. Cavum trigeminale) formed by two layers of the dura mater. Gassers's ganglion can be found near the apex of the petrous part of the temporal bone [14, 33, 41].

There is no consent among different authors, whether or not the V2 passes within the cavernous sinus. Some say that the V2 passes through the cavernous sinus embedded on its lateral wall [34]. However, other authors state that the V1 is the only part of the trigeminal nerve to reside within the cavernous sinus. After originating from the trigeminal ganglion the maxillary nerve courses beneath the dura of the middle fossa, below the level where the medial and lateral walls of the cavernous sinus join, at the lower edge of the ophthalmic nerve [14]. The V2 exits the skull through the foramen rotundum and enters into the sphenopalatine fossa. There it gives off several branches including the meningeal branches, the superior alveolar nerves, the zygomatic and infraorbital nerves. The nasal and palatine nerves originate from the pterygopalatine (sphenopalatine) ganglion. After passing through the pterygopalatine fossa the V2 enters the orbit through the inferior orbital fissure as the infraorbital nerve. Next, it courses through the infraorbital groove and canal in the orbit floor. Through the infraorbital foramen it emerges on the face between the levator labii superioris and the levator anguli oris muscles. Here it terminates by dividing into palpebral, nasal and superior labial branches [14, 27, 33, 34, 41]. Overall, the branches of the maxillary nerve can be divided into four groups depending on the place of origin i.e. [14]:

- In the cranium (meningeal nerve);

- In the sphenopalatine fossa (ganglionic branches, zygomatic nerve, posterior superior alveolar nerve, sphenopalatine ganglion, greater and lesser palatine nerves, nasal branches, nasopalatine nerve, pharyngeal nerve);

- In the infraorbital canal (middle and anterior superior alveolar nerves);

- On the face (inferior palpebral branches, nasal branches, superior labial branches).

There is not much literature data regarding the variations of the maxillary nerve. Siessere et al. [32] after dissecting 20 human heads observed no significant variations in relation to the anatomy of the $\mathrm{V} 2$. In the same specimens, the mandibular nerve had a $20 \%$ variation rate - showing high variability of the different branches of the trigeminal nerve $[17,27]$. The more common variation of the maxillary nerve include a bifid maxillary nerve, absent middle superior alveolar nerve, posterior superior alveolar nerve innervating areas normally covered by the long buccal nerve, and branches from the pterygopalatine ganglion supplying the abducent, optic or ciliary nerves [34].

\section{MAJOR BRANCHES OF THE MAXILLARY NERVE: THEIR COURSE AND VARIATION (FIG. 1)}

\section{Zygomatic nerve}

The zygomatic nerve arises from the maxillary nerve in the pterygopalatine fossa, passes anteriorly, superiorly, and laterally to enter the orbit through the inferior orbital fissure [14]. On the lateral wall of 


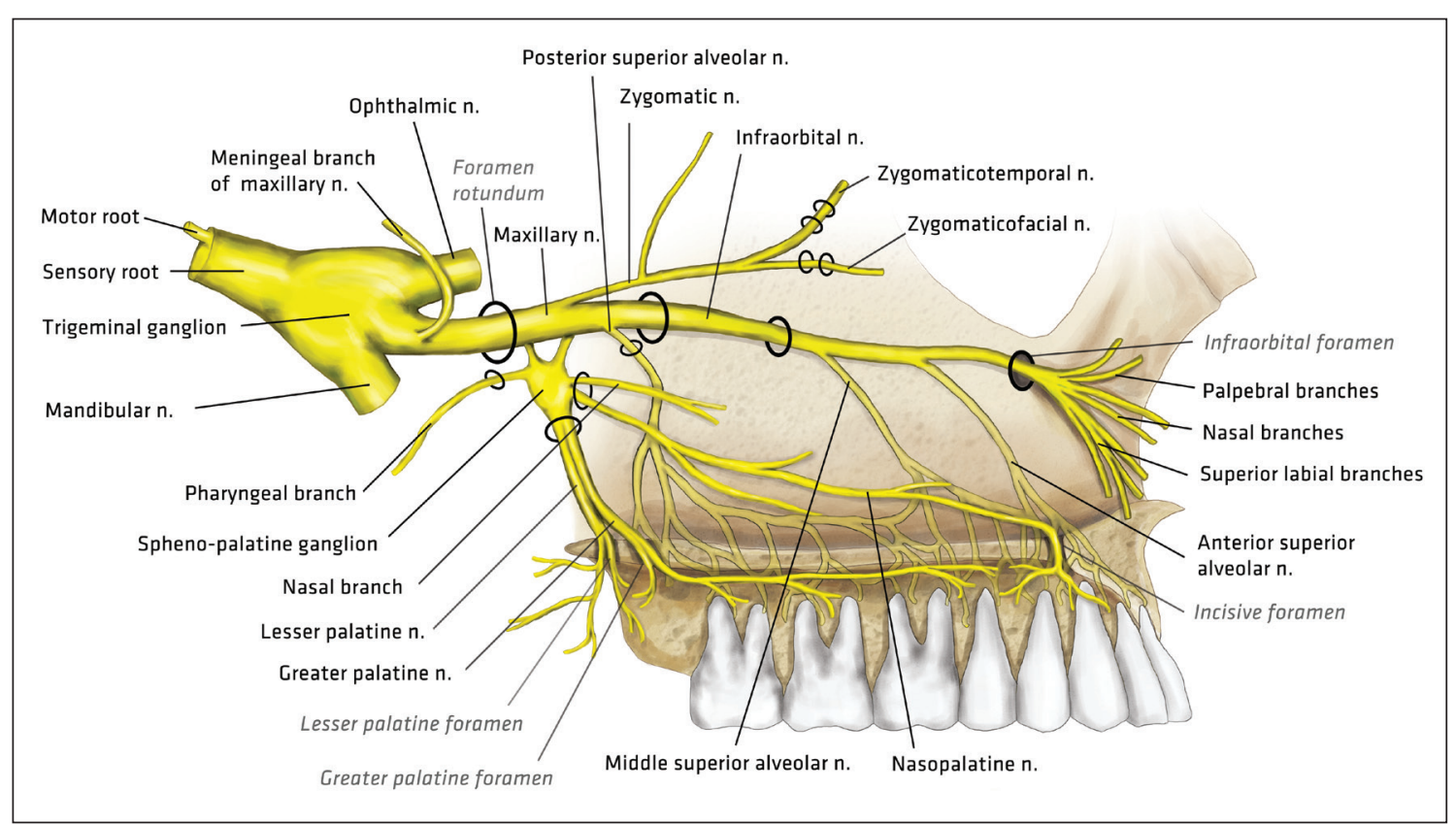

Figure 1. The maxillary nerve - origin, branches and their course. Schematic sagittal-section of a human head. The black circles represent foramina.

the orbit it divides into the zygomaticotemporal and zygomaticofacial branches. The zygomaticotemporal nerve courses along the inferolateral angle of the orbit where it communicates with the lacrimal nerve. At its terminus, the zygomaticotemporal nerve innervates the skin of the temporal area [38]. In cases where the lacrimal nerve is absent, the zygomaticotemporal nerve also innervates the lacrimal gland [38]. The zygomaticofacial nerve runs a similar rout to the zygomaticotemporal nerve but exists through the zygomaticofacial foramen of the zygomatic bone and supplies the skin on the prominence of the cheek. According to Moretto et al. [23], nearly $2 / 3$ of the extra-osseous trajectory of the maxillary nerve is located in the zygomatic region.

\section{Infraorbital nerve}

The infraorbital nerve is a direct continuation of the maxillary nerve. It runs anteriorly, inferior to the floor of the orbit, giving off superior alveolar nerve branches (posterior, middle and anterior superior alveolar nerves), which innervate the maxillary teeth. After exiting through the infraorbital foramen, it divides into 4 branches - the inferior palpebral, the external nasal, the internal nasal and the superior labial branches for the sensory innervation to the skin of the eyelid, nose, cheek and upper lip. Though usually single, the number of the infraorbital foramina can vary (i.e. 2-3) [1, 3, 8, 16, 39]. Aziz et al. [1] found a $15 \%$ incidence of accessory infraorbital foramina, Bressan et al. [3] reported this number to be $4.7 \%$ (higher frequency on the left side), while Gupta [8] discovered that only $1.3 \%$ of the analysed skulls had an accessory infraorbital foramen. Tubbs et al. [39] during a cadaveric dissection of a male discovered an accessory infraorbital foramen associated with a bifid infraorbital nerve. The distance from the infraorbital foramen to the inferior border of the orbital rim varies from 4.6 to $14 \mathrm{~mm}[1,4,15]$. Such differences pose a danger during procedures involving facial flaps [4].

\section{Posterior superior alveolar nerve}

The posterior superior alveolar nerve branches off the maxillary nerve before it enters the infraorbital groove. It descends on the maxillary tuberosity innervating on its way the gingiva and the mucosa of the cheek. Next, it enters the posterior alveolar canal and gives off branches to the membrane of the maxillary sinus and the molar teeth [18]. Murakami et al. [24] observed the posterior superior alveolar nerve passing through canaliculi in the lateral wall of the maxillary sinus in $62.2 \%$ of cases, or under the mucous membrane of the sinus in $37.8 \%$ cases

In cases, when the middle superior alveolar nerve is absent, the premolar teeth are also innervated by the posterior superior alveolar nerve $[11,18]$. The number of posterior superior alveolar nerves can vary from single to multiple. McDaniel [21] found that the 


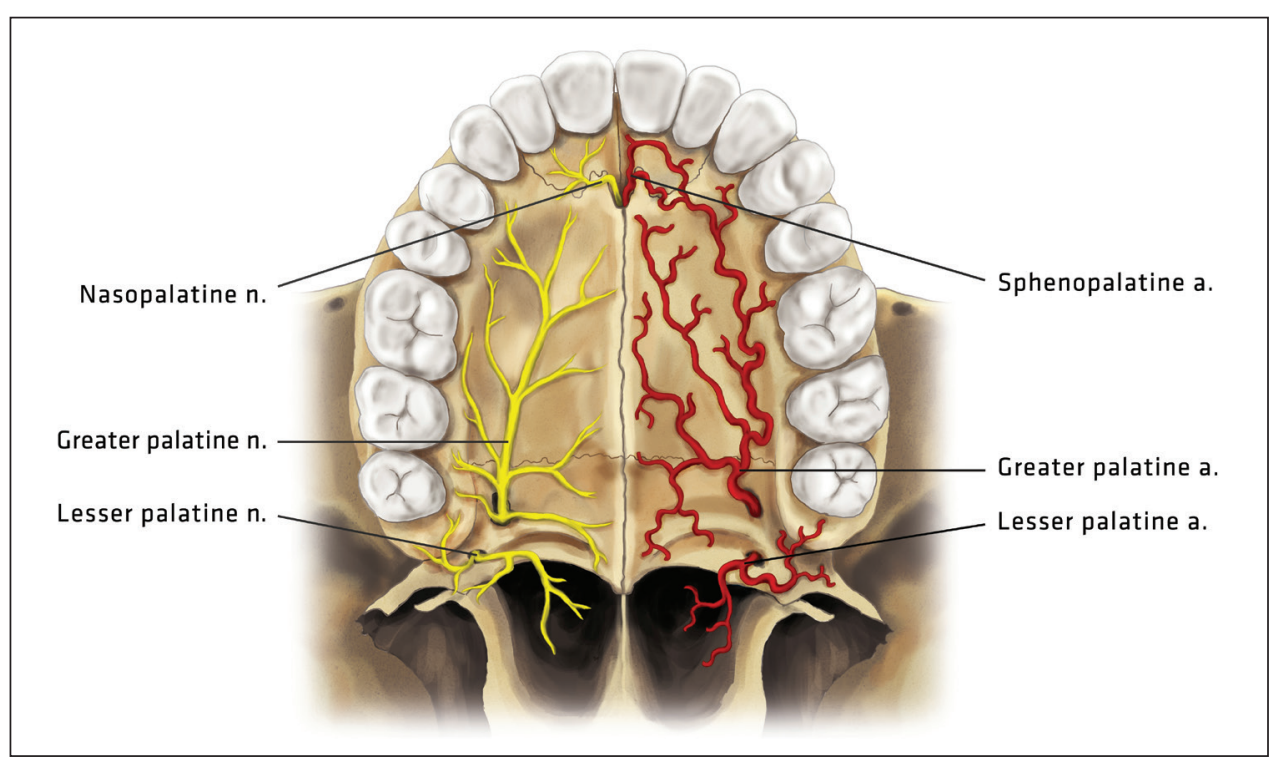

Figure 2. The palatine nerves and arteries — course and branch distribution on the palate.

posterior superior alveolar nerve had one branch in $21 \%$, two branches in $30 \%$ and three branches in $25 \%$ of specimens. On reaching the molar teeth, the posterior superior alveolar nerve gives rise to four groups of terminal branches [23]:

- Dental branches, to supply the molar and premolar roots;

- Alveolar branches, to the alveolar periosteum and gingival mucosa;

- Mucous branches, to supply the maxillary sinus mucosa:

- Bone branches to supply the maxilla.

\section{Middle superior alveolar nerve}

If present, the middle superior alveolar nerve branches off the infraorbital nerve in the infraorbital canal. It runs in the lateral wall of the maxillary sinus and supplies the premolar teeth [11]. McDaniel [21] reported that in only $30 \%$ of the examined cases the middle superior alveolar nerve its normal course. The majority of specimens presented with a nerve plexus (branches from either the posterior, anterior or both mentioned superior alveolar nerves) supplying the teeth rather than a unified nerve.

\section{Anterior superior alveolar nerve}

The anterior superior alveolar nerve leaves the infraorbital nerve in the orbital groove - the posterior part of the infraorbital canal [14, 33]. It enters the anterior wall of the maxillary sinus and there divides into branches supplying the incisor and canine teeth.
It runs further towards the canine fossa branching and forming the superior dental plexus (with the branches of the middle alveolar nerve if present) located in the maxillary alveolar process. From this plexus the nasal branches arise, and after passing through a small canal in the lateral wall of the inferior meatus, supply the mucous membrane of the anterior area of the lateral wall and the floor of the nasal cavity [30]. According to McDaniel [21] the anterior superior alveolar nerve has the form of a single trunk in $75 \%$ of cases, and in $25 \%$ it forms a small plexus from the nerve's terminal branches, which overlays the canine fossa. The plexus form seems to be favoured by multiple posterior branches, and by the presence of a middle branch or an anterior branch with multiple main branches [27].

\section{Palatine nerves (Fig. 2)}

Both palatine nerves - the greater and the lesser palatine nerve - originate from the sphenopalatine ganglion. The greater palatine nerve descends through the greater palatine canal (mean canal length $31 \mathrm{~mm}$, range 15-44 mm) $[35,36]$, and the greater palatine foramen to run anteriorly in a groove on the inferior surface of the hard palate. It innervates the hard palate and the palatal gingiva. The thickest nerves connecting the sphenopalatine ganglion are the sphenopalatine nerve and the greater palatine nerve [28]. The lesser palatine nerve (or nerves) descends through the lesser palatine foramen (or foramina) (LPF), and through its branches innervates the uvula, tonsil, and the soft palate. These nerves anastomose with branches of 
the glossopharyngeal nerve to form a tonsillar plexus around the palatine tonsil [30]. Our recent [37] large original study on dry skulls and head computed tomography's, combined with a meta-analysis of all the previous observational studies on the subject of the location of the greater palatine foramen (GPF), has shown that the pooled prevalence of the GPF being positioned opposite the third maxillary molar (M3) is $63.9 \%(95 \% \mathrm{Cl}=56.6-70.9 \% ; \mathrm{n}=6,927 \mathrm{GPF})$. In the Polish sample ( $n=2,700$ GPF), the GPF was located opposite the M3 in $74.7 \%$, opposite the $\mathrm{M} 2$ in $16.3 \%$, and in $6.8 \%$ between the $M 2$ and the M3. The number of LPF varied from 0 to $5[9,13,26,31]$ showing that in some cases the lesser palatine nerve can exit on the palate through the GPF. This makes it prone to anaesthesia when blocking the greater palatine nerve. Also a high number of LPF may lead to the formation of a single large LPF, as Jaffar and Hamadah [13] have found. Such an anatomical variation may lead to mistaking the LPF for the GPF, and thus anaesthetising the lesser palatine nerve, leading to a gagging sensation in the soft palate [9]. The nerve supply of the greater palatine nerve can also vary, as it sometime gives off branches to innervate the molar and premolar teeth [27].

\section{Nasopalatine nerve (Fig. 2)}

The nasopalatine nerve is the largest of the nasal branches originating from the sphenopalatine ganglion. It enters the nasal cavity through the sphenopalatine foramen, and reaches the nasal septum. Running anteriorly and inferiorly between the periosteum and the mucous membrane of the nasal septum it innervates the nasal septum filaments. Further on its course it exits the nasal cavity through the incisive foramen. Its terminal branches innervate the mucosa of the anterior part of the hard palate, and the palatal gingiva near the canine teeth. These branches may communicate with the branches of the greater palatine nerve $[14,30]$. In some cases the nasopalatine nerve can innervate the incisor teeth [22], thus making the nasopalatine nerve block necessary to complete the anaesthesia of the central incisor [27].

\section{MAXILLARY NERVE ANAESTHESIA AND RELATED CLINICAL SIGNIFICANCE OF MAXILLARY NERVE BRANCHES}

\section{Maxillary nerve anaesthesia}

Hawkins and Isen [10] describe two intra-oral techniques of blocking the maxillary nerve - the high tuberosity and the greater palatine canal approach.
The latter is associated with a higher success rate and a lower incidence of complications [40]. However, the major clinical difficulty of this method is to accurately locate the position of the GPF [40]. On the other hand, the high tuberosity approach is easier for dentists, as it is essentially a high buccal infiltration [34]. However, complications may include a lack of profound anaesthesia, and a high risk of producing a hematoma from puncturing the pterygoid venous plexus $[10,40]$.

\section{Greater palatine nerve and nasopalatine nerve block}

This block is produced at the site where the greater palatine nerve exists on the palate. The GPF is most often located opposite the third maxillary molar. The nasopalatine nerve enters the palate through the incisive foramen, and can be clinically located through the position of the incisive papilla. Different areas of the palate can be anaesthetised by local infiltration into the papillae, especially if the procedure involves only one or two teeth [19]. During this procedure the clinician has to bear in mind the fact that the lesser palatine nerve exits in the vicinity of the GPF and its anaesthesia can lead to a gagging sensation in the soft palate, as mentioned earlier [9].

\section{Posterior superior alveolar nerve block}

This block is commonly used to produce anaesthesia in the maxillary molars. Freuen et al. [7] suggest this block to anesthetize the maxillary molars with the exception of the mesiobuccal root of the first maxillary molar, as this structure can be innervated by the middle superior alveolar nerve [19]. In such a case a second injection is warranted in $28 \%$ of patients [34]. Another way to deal with such a case is to add to the posterior superior alveolar nerve block a greater palatine nerve block to enhance the anaesthetic effect [2]. The most common complication is a haematoma resulting from puncturing the pterygoid plexus. Other uncommon complications include temporary Bell's palsy, injury of the parotid gland, and trismus [34]. The different origins of the posterior superior alveolar nerve compared with the middle and the anterior branches offers the possibility to anesthetise only the posterior branch.

\section{Middle superior alveolar nerve block}

A middle superior alveolar nerve block injection should be administered if the infraorbital nerve block does not provide adequate anaesthesia to the teeth distal of the canine, or if the posterior superior alve- 
olar injection does not provide anaesthesia for the mesiobuccal root of the first molar [34].

\section{Infraorbital nerve block}

This technique allows for producing anaesthesia from the maxillary central incisor to the premolar teeth in 70\% of patients [34]. Through one injection, the clinician can anaesthetize the anterior and middle superior alveolar nerves, as well as the terminal branches of the infraorbital nerve. McDaniel [21] states that an infraorbital or zygomatic nerve block, or both, produce anaesthesia in all maxillary teeth. Salomão et al. [29] proposed a technique for producing the infraorbital nerve block, which uses the superior lateral labial fraenum as an anatomic reference point. The constancy of this point, independent of the presence or absence of teeth, is considered as the main advantage of this technique.

\section{Supraperiosteal injection}

This field block allows anaesthetizing chosen terminal branches of the maxillary nerve. It is used for pulpal and soft tissue anaesthesia. Infection or increased bone density can diminish or abolish the anaesthetic effect.

\section{Trigeminal neuralgia}

When writing about the maxillary nerve, it is worth to mention trigeminal neuralgia, as the maxillary and mandibular nerves are the ones that most often transmit pain in this disorder [25]. The reason behind this neuralgia is vascular compression of the nerves by vessel loops or aneurysms [5]. The higher prevalence of right-sided pain in this disorder can be explained by the fact that the rotundum and ovale foramina on the right side of the human cranium are significantly narrower than on the left side [25]. This can lead to easier compression of the exiting nerves.

\section{CONCLUSIONS}

This review summarises the data on the anatomy and variations of the maxillary nerve and its branches. The mentioned variations are often the cause of anaesthesia failure or surgical complications. A thorough understanding of the anatomy will allow for careful planning and execution of anaesthesiological and surgical procedures involving the maxillary nerve and its branches.

\section{ACKNOWLEDGEMENTS}

The authors of this manuscript wish to thank Ms Karolina Saganiak for her excellent illustrations.

\section{AUTHOR CONTRIBUTION}

Design and planning of the review - IMT, JAW. Bibliographic search - IMT, HZ, TG. Drafting and revising the manuscript - IMT, HZ. Critical revision of the manuscript - TG, JAW. All authors have read and approved the final version of the manuscript.

All co-authors confirm the above-mentioned contributions and consent to the fact that this manuscript is a part of Iwona M. Tomaszewska's PhD thesis. The co-authors confirm that Iwona M. Tomaszewska has contributed significantly ( $80 \%$ in total) to every part of this paper, as stated above.

\section{REFERENCES}

1. Aziz SR, Marchena JM, Puran A (2000) Anatomic characteristics of the infraorbital foramen: a cadaver study. J Oral Maxillofac Surg, 58: 992-996.

2. Blanton PL, Jeske AH (2003) The key to profound local anesthesia: neuroanatomy. J Am Dent Assoc, 134: 753-760.

3. Bressan C, Geuna S, Malerba G, Giacobini G, Giordano M, Robecchi MG, Vercellino V (2004) Descriptive and topographic anatomy of the accessory infraorbital foramen. Clinical implications in maxillary surgery. Minerva Stomatol, 53: 495-505.

4. Chandra RK, Kennedy DW (2004) Surgical implications of an unusual anomaly of the infraorbital nerve. Ear Nose Throat J, 83: 766-767.

5. Dzierżanowski J, Słoniewski P (2014) Trigeminal neuralgia caused by aneurysm of the posterior cerebral artery: a case description and the analysis of anatomical variety of vascular complex in the rood entry zone of trigeminal nerve. Folia Morphol, 73: 224-228.

6. Falconer MA (1949) Intramedullary trigeminal tractotomy and its place in the treatment of facial pain. J Neurol Neurosurg Psychiat, 12: 297-311.

7. Freuen ND, Feil BA, Norton NS (2007) The clinical anatomy of complications observed in a posterior superior alveolar nerve block. FASEB J, 21: 776.4 .

8. Gupta T (2008) Localization of important facial foramina encountered in maxillo-facial surgery. Clin Anat, 21: 633-640.

9. Hassanali J, Mwaniki D (1984) Palatal analysis and osteology of the hard palate of the Kenyan African skulls. Anat Rec, 209: 273-280.

10. Hawkins JM, Isen D (1998) Maxillary nerve block: the pterygopalatine canal approach. Calif Dent Assoc, 26: 658-664.

11. Heasman PA (1984) Clinical anatomy of the superior alveolar nerves. Br J Oral Maxillofac Surg, 22: 439-447.

12. Higashiyama H, Kuratani S (2014) On the maxillary nerve. J Morphol, 275: 17-38.

13. Jaffar AA, Hamadah HJ (2003) An analysis of the position of the greater palatine foramen. J Basic Med Sci, 3: 24-32.

14. Joo W, Yoshioka F, Funaki T, Mizokami K, Rhoton AL Jr (2014) Microsurgical anatomy of the trigeminal nerve. Clin Anat, 27: 61-88.

15. Kazkayasi M, Ergin A, Ersoy M, Tekdemir I, Elhan A (2003) Microscopic anatomy of the infraorbital canal, nerve, and foramen. Otolaryngol Head Neck Surg, 129: 692-697. 
16. Leo JT, Cassell MD, Bergman RA (1995) Variation in human infraorbital nerve, canal and foramen. Ann Anat, 177: 93-95.

17. Lipski M, Tomaszewska IM, Lipska W, Lis GJ, Tomaszewski KA (2013) The mandible and its foramen: anatomy, anthropology, embryology and resulting clinical implications. Folia Morphol, 72: 285-292.

18. Loetscher CA, Walton RE (1988) Patterns of innervation of the maxillary first molar: a dissection study. Oral Surg Oral Med Oral Pathol, 65: 86-90.

19. Malamed SF, Trieger N (1983) Intraoral maxillary nerve block: an anatomical and clinical study. Anesth Prog, 30: 44-48.

20. Manni E, Bortolami R, Deriu PL (1970) Presence of cell bodies of the afferents from the eye muscles in the semilunar ganglion. Arch Ital Biol, 108: 106-120.

21. McDaniel WM (1956) Variations in nerve distributions of the maxillary teeth. J Dent Res, 35: 916-921.

22. Meyer TN, Lemos LL, Nascimento CN, Lellis WR (2007) Effectiveness of nasopalatine nerve block for anesthesia of maxillary central incisors after failure of the anterior superior alveolar nerve block technique. Braz Dent J, 18: 69-73.

23. Moretto EP, Silva GH, Toledo Filho JL, Andreo JC, Navarro Rde L, Navarro JA (2005) Surgical anatomy of the maxillary nerve in the zygomatic region. J Appl Oral Sci, 13: 167-170.

24. Murakami G, Ohtsuka K, Sato I, Moriyama H, Shimada K, Tomita H (1994) The superior alveolar nerves: their topographical relationship and distribution to the maxillary sinus in human adults. Okajimas Folia Anat Jpn, 70: 319-328.

25. Neto HS, Camilli JA, Marques MJ (2005) Trigeminal neuralgia is caused by maxillary and mandibular nerve entrapment: greater incidence of right-sided facial symptoms is due to the foramen rotundum and foramen ovale being narrower on the right side of the cranium. Med Hypotheses, 65: 1179-1182.

26. Piagkou M, Xanthos T, Anagnostopoulou S, Demesticha T, Kotsiomitis E, Piagkos G, Protogerou V, Lappas D, Skandalakis P, Johnson EO (2012) Anatomical variation and morphology in the position of the palatine foramina in adult human skulls from Greece. J Craniomaxillofac Surg, 40: e206-e210.

27. Rodella LF, Buffoli B, Labanca M, Rezzani R (2012) A review of the mandibular and maxillary nerve supplies and their clinical relevance. Arch Oral Biol, 57: 323-334.

28. Rusu MC, Pop F, Curca GC, Podoleanu L, Voinea LM (2009) The pterygopalatine ganglion in humans: a morphological study. Ann Anat, 191: 196-202.

29. Salomão JI, Salomão JA, Salomão Costa RC (1990) New anatomic intraoral reference for the anesthetic blocking of the anterior and middle maxillary alveolar nerves (infraorbital block). Braz Dent J, 1: 31-36.

30. Shankland WE $2^{\text {nd }}(2001)$ The trigeminal nerve. Part III: The maxillary division, Cranio, 19: 78-83.

31. Sharma NA, Garud RS (2013) Greater palatine foramen: key to successful hemimaxillary anaesthesia: a morphometric study and report of a rare aberration. Singapore Med J, 54: 152-159.

32. Siéssere $S$, Hallak Regalo SC, Semprini M, Honorato De Oliveira R, Vitti M, Mizusaki lyomasa M, Mardegan Issa JP, De Sousa LG (2009) Anatomical variations of the mandibular nerve and its branches correlated to clinical situations. Minerva Stomatol, 58: 209-215.

33. Soeira G, Abd el-Bary TH, Dujovny M, Slavin KV, Ausman JI (1994) Microsurgical anatomy of the trigeminal nerve. Neurol Res, 16: 273-283.

34. Somayaji KS, Rao MK (2012) Anatomy and clinical applications of the maxillary nerve in dentistry: a literature review. Dent Update, 39: 727-730, 733-735.

35. Tomaszewska IM, Frączek P, Gomulska M, Pliczko M, Śliwińska A, Sałapa K, Chrzan R, Kowalski P, Nowakowski M, Walocha JA (2014) Sex determination based on the analysis of a contemporary Polish population's palatine bones - a computed tomography study of 1200 patients. Folia Morphol, 73: 462-468.

36. Tomaszewska IM, Kmiotek EK, Pena IZ, Średniawa M Czyżowska K, Chrzan R, Nowakowski M, Walocha JA (2014) Computed tomography morphometric analysis of the greater palatine canal - a study of 1500 head CTs and a systematic review of literature. Anat Sci Int, DOI: 10.1007/s12565-014-0263-9.

37. Tomaszewska IM, Tomaszewski KA, Kmiotek EK, Pena IZ, Urbanik A, Nowakowski M, Walocha JA (2014) Anatomical landmarks for the localization of the greater palatine foramen: a study of 1200 head CTs, 150 dry skulls, systematic review of literature and meta-analysis. J Anat, 225: 419-435.

38. Totonchi A, Pashmini N, Guyuron B (2005) The zygomaticotemporal branch of the trigeminal nerve: an anatomical study. Plast Reconstr Surg, 115: 273-277.

39. Tubbs RS, Loukas M, May WR, Cohen-Gadol AA (2010) A variation of the infraorbital nerve: its potential clinical consequence especially in the treatment of trigeminal neuralgia: case report. Neurosurgery, 67: onsE315; discussion onsE315.

40. Wong JD, Sved AM (1991) Maxillary nerve block anaesthesia via the greater palatine canal: a modified technique and case reports. Aust Dent J, 36: 15-21.

41. Zheng JP, Song $M$, Zhan $X X$, Li CZ, Zong $X Y$, Zhang $Y Z$ (2014) Endoscopic approach to the trigeminal nerve: An anatomic study. J Craniomaxillofac Surg, 42: 674-682. 